\title{
A novel dual antibody conjugate (Heteropolymer) reduces circulating HIV in a mouse model SE Pincus*1, SM Jones ${ }^{1}$, TA Bradley ${ }^{1}$, J Dunne ${ }^{1}$, Q Wang1, WJ Honnen ${ }^{2}$, R Kunert ${ }^{3}$ and A Pinter ${ }^{2}$
}

\author{
Address: ${ }^{1}$ Elusys Therapeutics, Pine Brook, NJ 07058, USA, 2Public Health Research Institute, Newark, NJ 07103, USA and ${ }^{3}$ Institute of Applied \\ Microbiology, Vienna, Austria \\ * Corresponding author
}

\author{
from 2006 International Meeting of The Institute of Human Virology \\ Baltimore, USA. 17-2I November, 2006 \\ Published: 21 December 2006 \\ Retrovirology 2006, 3(SuppI I):P76 doi:I0.II86/1742-4690-3-SI-P76
}

(C) 2006 Pincus et al; licensee BioMed Central Ltd.

HIV resistance to approved therapeutics is an increasing concern necessitating the need for development of new therapeutic approaches. Elusys's novel Heteropolymer (HP) technology consists of two monoclonal antibodies that are chemically cross-linked: one specific for complement receptor type 1 (CR1, CD35) on red blood cells (RBCs) and the other recognizing the target pathogen. Previous studies with multiple families of viruses have demonstrated that administration of HPs leads to rapid immobilization of virus to RBCs followed by delivery to the Kupffer cells of the liver where the viruses were destroyed.

Two different HIV HPs with distinct gp120 binding specificities were generated and shown to simultaneously bind to transgenic mouse (TgN) RBCs (expressing human CR1) and gp120 in vitro by FACS analysis. Both HIV HPs were able to reduce the circulation in plasma of inactivated HIV more rapidly than the corresponding HIV Mabs in the TgN mouse. Both HIV HPs neutralized HIV strains SF162 and NL4-3 while only HIV HP 2 was able to neutralize SHIV89.6P.

These results support Elusys continuing efforts and testing of HIV HP as a new therapeutic approach. 